\title{
Muscle Roles on Directional Change during Hopping of a Biomimetic Feline Hindlimb
}

\author{
Andre Rosendo, Kenichi Narioka and Koh Hosoda
}

\begin{abstract}
Cats, from tiny domestic cats (Felix Catus) to big tigers (Panthera Tigris), are well known for their great acrobatic skills and hunting ability. Aiming to better understand how the feline family interacts with the environment, we adopt a biomimetic approach on a hopping feline hindlimb. Using air muscles to simulate the compliance of biological muscles, this robotic hindlimb has seven muscles and changes hopping direction. We individually evaluate and estimate muscles contribution to the jumping direction. Finally, we successfully control the hopping direction using a non-linear curve fitting from experimental results, hopefully contributing to the understanding of our biological counterpart.
\end{abstract}

\section{INTRODUCTION}

Members from the Felidae family have always amazed researchers for being the pinnacle of landed animal's locomotion. Abilities such as tree climbing, soft landing and high speed locomotion are some of their feats which baffle biologists and robotic researchers. For more than 100 years, researchers have been trying to pinpoint the secret for such intriguing proficiency, but due to highly complex and redundant muscular structures, this biological conundrum, so far, have not been fully explained or mimicked.

With the advent of electromyographic (EMG) sensors, improved image capturing and digital computers, biologists could deepen their knowledge on musculoskeletal behavior of animals. Great works that could be mentioned would be Engberg and Lundberg [1], Goslow [2] [3], and English [4].These works used EMG and biometric data to understand muscular activation pattern within the feline gait, reaching the conclusion that quadruped step cycle is divided in four phases and, independent of gait pattern or speed, is controlled by 3 muscular patterns.

Although these works helped understanding overall feline musculoskeletal behavior, particular muscular function (i.e. isolated from the redundancy of dozens of muscles in vivo) was still very hard to understand. Light started to be shed on this problem with the approach of mechanical energy transfer done by the so called biarticular muscles [5] [6], which has as its foremost researcher van Ingen Schenau (e.g., [7] and [8]). These muscles were proven to contribute to energy transfer between proximal and distal joints, keeping coordination between joints, thus helping on the final direction of the external force [9].

Drawing inspiration from biological systems, roboticists have been trying to mimic animal locomotion for some

This work was partially supported by KAKENHI Kiban(S) 23220004

The authors are with Multimedia Department, Faculty of Information Science and Technology, Osaka University, 1-5 Yamadaoka, Suita, Osaka, Japan. andre.rosendodist.osaka-u.ac.jp time. Considering geometrical resemblance we could mention Tekken 2 and Oncilla, which are controlled by electrical motors and have a passive element at each leg to soften the contact with the floor [10] [11]. Although these robots have remarkable performance, with stable locomotion along different gait patterns, their robotic actuation system differs drastically from biological systems.

Due to this fundamental difference, the development of a robotic hindlimb which faithfully represents the biological system should possess some degree of actuation and compliance in every joint, as observed in muscles. In this aspect a few robots could be mentioned, such as Puppy [12], which is a canine robot driven by pneumatic muscles, capable of drawing power while being elastic, similarly to the pneumatic robot created by Tsujita [13]. Another example would be the Cheetah robot [14], which combines electric motor and pneumatic cylinder inside the same muscle, possessing a simplified structure of only 2 muscles per leg. Also worth citing is the robot Kenken [15], which had a spring simulating the behavior of the muscle gastrocnemius. Although closer to a biological representation, these four robots had over-simplified muscular structure, not allowing mono and biarticular role studies.

To the best of the authors knowledge, works that better represented artificially this biological complexity, including mono and biarticular muscles, were the works of Ekeberg [16], Niiyama [17] and Hosoda [9]. While Hosoda and Niiyama focused on human locomotion during jumping and running, with Hosoda investigating specifically biarticular muscle roles during jumping, Ekeberg developed a feline walking computer simulation, capable of adapting to terrain irregularities.

In this paper, we attack the animal locomotion problem with a more biologically faithful approach. Adopting a hindlimb structure similar to the one explored by Ekeberg, we evaluated the contribution of mono and biarticular muscles during directional change of a feline jump while attached to a slider.

The robotic hindlimb is tested during hopping with different settings for gastrocnemius, semitendinosus and iliopsoas, and their contribution to the landing position is evaluated. Our hindlimb is capable of changing hopping direction by changing the level of air pressure at the semitendinosus muscle, being controlled by a non-linear relationship. In the future, this same method will be applied to a full body robot, inducing controlled bounding and pronking behaviors, hopefully solving remaining questions on animal locomotion. 
TABLE I

PNEUPARD'S HINDLIMB KEY CHARACTERISTICS

\begin{tabular}{c|c}
\hline Property & Value \\
\hline Thigh length & $287 \mathrm{~mm}$ \\
\hline Shank length & $345 \mathrm{~mm}$ \\
\hline Foot length & $132 \mathrm{~mm}$ \\
\hline Body weight & $870 \mathrm{grams}$ \\
\hline Hindlimb weight & 450 grams \\
\hline Total weight & $1.32 \mathrm{~kg}$ \\
\hline
\end{tabular}

\section{MATERIALS AND METHODS}

In order to better understand the feline hindlimb, and later apply this muscular knowledge to any other animal, we believe that a constructivist approach is needed. Instead of observing the muscular behavior of a cat and trying to speculate how dozens of muscles are correlated, we believe that artificially replicating the same morphology and inducing the contraction of the muscles under investigation can produce more fruitful results, as discussed in [9].

For this research, due to physical constraints from air muscles (short muscles are unfeasible), a hindlimb of a sufficiently big cat had to be chosen. Considering its jumping proficiency, we adopted as biological model a Panthera Pardus, which will be integrated in the future with a full body pneumatic robot, hence named Pneupard. The specifications for the hindlimb can be found on table I, while the picture of the same attached to the slider on Fig. 1.

The attachment between the hindlimb and slider allows body rotation over sagittal plane, where this rotation axis is aligned with the hip rotation axis. Due to the non-existence of digits, the hindlimb touches the floor with the tip of its metatarsal link (foot).

Similarly to the computational model adopted by Ekeberg [16], the adopted hindlimb possesses 7 muscles, with 5 monoarticular muscles performing ankle flexion (tibialis anterior, TA), ankle extension (soleus, SO), knee (stifle) extension (vastus lateralis, VL), hip flexion (iliopsoas, IP) and hip extension (anterior biceps, AB), and 2 biarticular muscles performing knee flexion and ankle extension (gastrocnemius, GA) and knee flexion and hip extension (semitendinosus, $\mathrm{ST}$ ). We adopt pneumatic muscles as biological muscles replacements, and their output force obeys the following relationship:

$$
F \propto \frac{p_{\text {air }}}{\Delta l / L_{0}}
$$

where $F$ is the output force, $p_{\text {air }}$ is the pressure inside the air muscle, $L_{0}$ is the relaxed length and $\Delta l$ is the length difference after muscular activation, as seen in [18]. Thus, the amount of pressure can be directly related to the degree of muscular tonus (power) and small muscular deformation (small $\Delta l$ ) results in high power (due to the higher elastic potential energy associated).

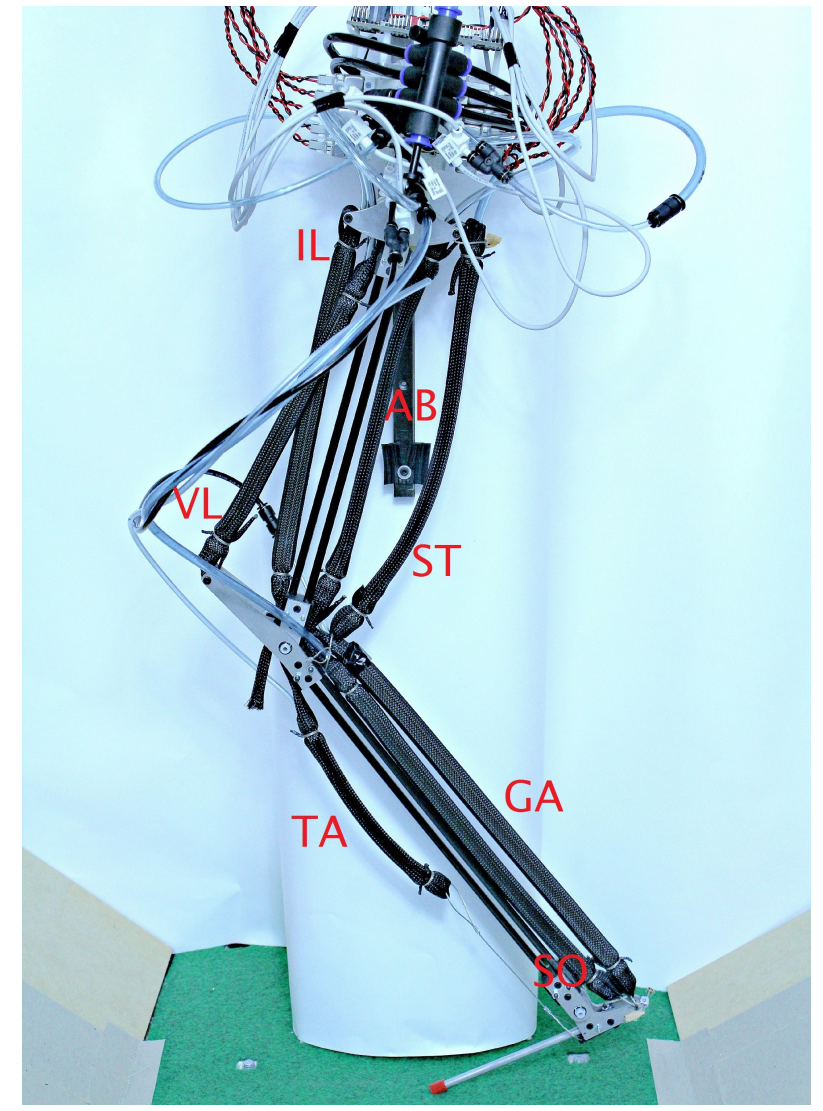

Fig. 1. Feline hindlimb attached to slider. Vertical translation and sagittal rotation are possible between the hip and the slider.

\section{A. Muscular Activation and Pattern}

The muscular activation is done by pilot-operated onoff pneumatic valves. Instead of a "bang-bang" approach for muscular contraction, we opted for a hysteresis control approach, being capable of controlling the air pressure inside each muscle. The maximum pressure used for jumping was $0.6 \mathrm{MPa}$, and the aforementioned control method allows incremental pressure changes of $0.01 \mathrm{MPa}$.

The hindlimb is controlled through a microcontroller, attached to its body and connected to a computer. The microcontroller, called Adaptive Board and created by our Adaptive Robotics Laboratory, runs an ARM $76 \mathrm{MHz}$ processor and samples data from the hindlimb at a frequency of $1 \mathrm{kHz}$, transmitting to the computer at a frequency of $40 \mathrm{~Hz}$. A tether connected to the hindlimb allows communication, along with supplying energy and compressed air.

The hopping pattern adopted for Pneupard's hindlimb is largely based on the jumping pattern demonstrated in [9], but with crucial differences: Since this work focus on a feline hindlimb, a digitigrade stance is possible by a higher torque on the ankle, while Hosoda's work focused on a plantigrade human morphology. Moreover, our robot starts jumping from the ground, instead of falling from a higher position.

From the initial position we control the muscles GA, ST and IL to reach a desired pressure. When these 3 parameters are fixed, the hindlimb jumps by supplying air to $\mathrm{SO}, \mathrm{AB}$ 


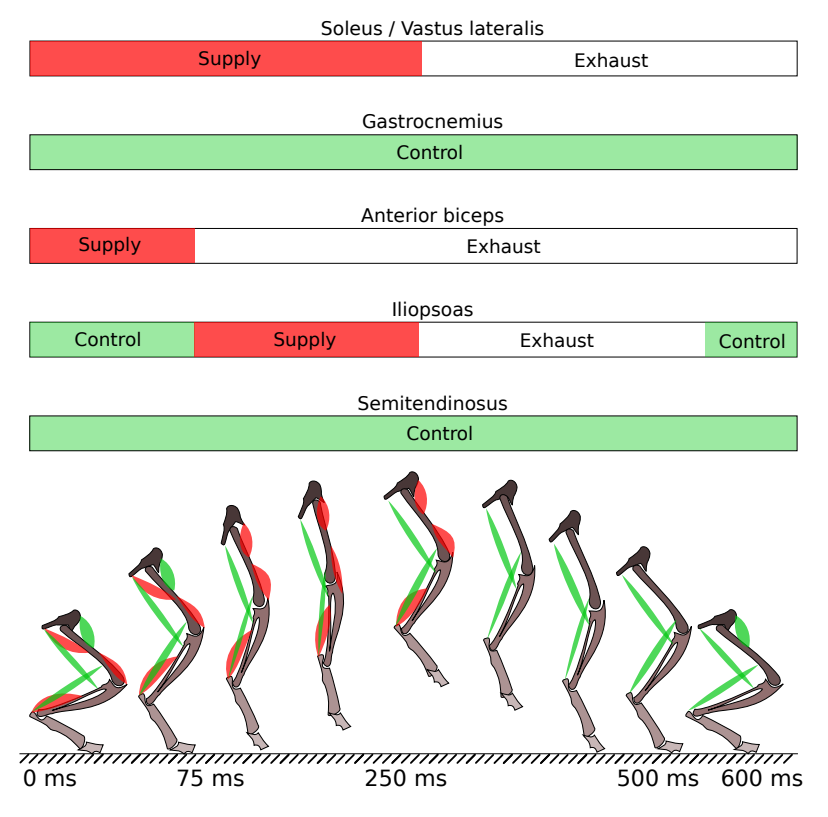

Fig. 2. Activation pattern of hindlimb, starting from ground position, reaching apex and landing.

and VL, which are the three main extensors, until the leg loses contact with the floor, where the muscle $\mathrm{AB}$ is relaxed (exhausted) and IL contracts even more. All monoarticular muscles are relaxed when the apex is reached (close to 250 $\mathrm{ms}$ ) to prepare for landing, where only the biarticular muscles absorb the impact. After impact, IL is once again regulated to reach the controlled value. A schematic demonstrating this activation pattern is shown in Fig. 2 .

\section{B. Role of Biarticular Muscles}

To understand the role of biarticular muscles, we elaborate an explanation from [8]. Similarly to van Ingen Schenau, we can depict the body acting against an external force, such as the ground reaction force (GRF) during landing in Fig. 3.

Notice that the total work done by the tip of the foot is the sum of works done by the other joints:

$$
\Delta W=T_{\text {hip }} \Delta \theta_{\text {hip }}+T_{\text {knee }} \Delta \theta_{\text {knee }}+T_{\text {ankle }} \Delta \theta_{\text {ankle }}
$$

Considering the frame of reference at the hip (Fig. 3B), the cartesian position of the foot would be defined as:

$$
\begin{aligned}
& x \\
& y
\end{aligned}=\begin{gathered}
-l_{\text {thigh }} \cos \left(\theta_{\text {hip }}\right)-l_{\text {shank }} \cos \left(\theta_{\text {hip }}-\theta_{\text {knee }}\right)-l_{\text {foot }} \cos \left(\theta_{\text {hip }}-\theta_{\text {knee }}+\theta_{\text {ankle }}\right) \\
-l_{\text {thigh }} \sin \left(\theta_{\text {hip }}\right)-l_{\text {shank }} \sin \left(\theta_{\text {hip }}-\theta_{\text {knee }}\right)-l_{\text {foot }} \sin \left(\theta_{\text {hip }}-\theta_{\text {knee }}+\theta_{\text {ankle }}\right)
\end{gathered}
$$

and for small displacements the relation between the changes in joint angles with changes at the tip of the foot would be:

$$
\left[\begin{array}{l}
\Delta x \\
\Delta y
\end{array}\right]=J\left[\begin{array}{c}
\Delta \theta_{\text {hip }} \\
\Delta \theta_{\text {knee }} \\
\Delta \theta_{\text {ankle }}
\end{array}\right]
$$

where $\mathrm{J}$ is the Jacobian, given by

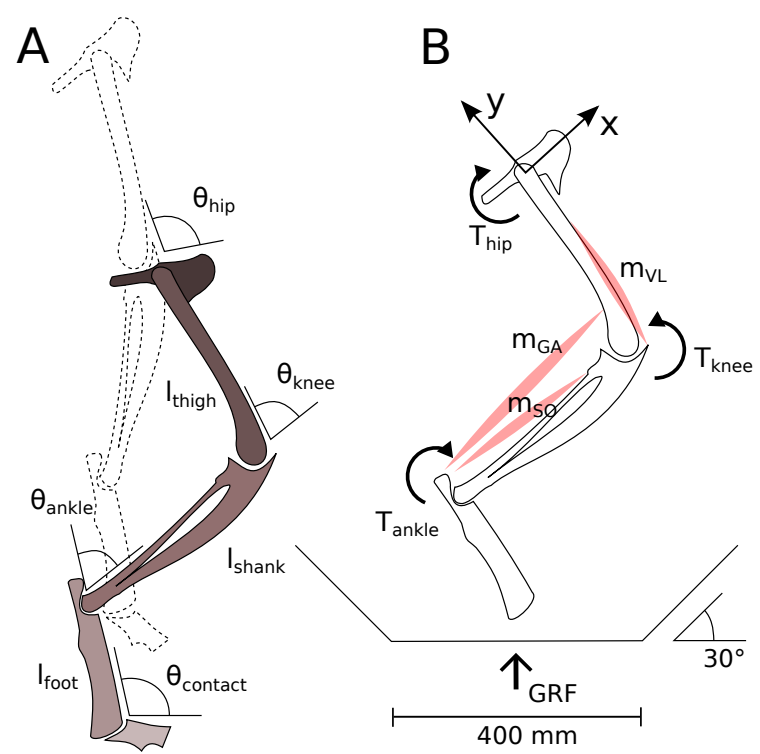

Fig. 3. Role of biarticular muscles, demonstrating relationship between GA, SO and VL.

$$
\begin{aligned}
& J=\left[\begin{array}{c}
l_{\text {thigh }} \sin \left(\theta_{h}\right)+l_{\text {shank }} \sin \left(\theta_{h}-\theta_{k}\right)+l_{\text {foot }} \cos \left(\theta_{h}-\theta_{k}+\theta_{a}\right) \\
-l_{\text {thigh }} \cos \left(\theta_{h}\right)-l_{\text {shank }} \cos \left(\theta_{h}-\theta_{k}\right)-l_{\text {foot }} \cos \left(\theta_{h}-\theta_{k}+\theta_{a}\right)
\end{array}\right.
\end{aligned}
$$

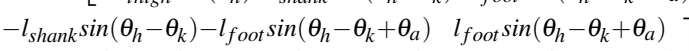

$$
\begin{aligned}
& \left.l_{\text {shank }} \cos \left(\theta_{h}-\theta_{k}\right)+l_{\text {foot }} \cos \left(\theta_{h}-\theta_{k}+\theta_{a}\right)-l_{\text {foot }} \cos \left(\theta_{h}-\theta_{k}+\theta_{a}\right)\right]
\end{aligned}
$$

Finally, the relationship between a change in the external force and a change in joint torques would be:

$$
\Delta T=J^{T} \Delta F
$$

Considering $T_{\text {ankle }}$ as a reaction to GRF generated by ankle extensors, activating biarticular muscles would be more effective than relying on monoarticulars. The reason for this comes from the tapered muscular structure that mammals usually possess, where power (muscle) is concentrated on proximal members, connecting to distal members with fewer muscles or tendon/sheath structures, such as the human hand. Transferring energy from the strong knee extensor VL through GA reduces the force needed on SO (as demonstrated in Fig 3B). The relationship between SO and GA was quantitatively approached in [5].

\section{Experimental Procedure}

We performed experiments by attaching the hindlimb on a linear slider, allowing the body to rotate along the sagittal plane. Due to horizontal translational constraints we added two slopes, each one $200 \mathrm{~mm}$ away from the slider's vertical projection, elevating 30 degrees to allow foot contact at steeper angles, as seen in Fig 3B.

At the first phase of the experiment the pressure at IL, GA and ST was fixed at a determined value and the hindlimb hopped 25 times. The landing position of each jump was recorded and new parameters for IL, GA and ST were set. Instead of recording the landing position with angular information, we judged that adopting distance would be more 


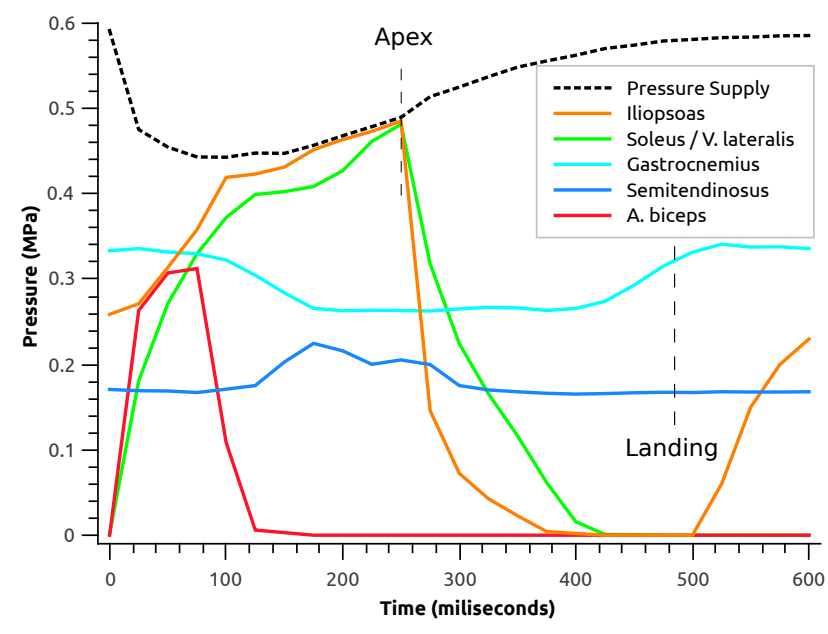

Fig. 4. Muscular activation (pressure) during hoping.

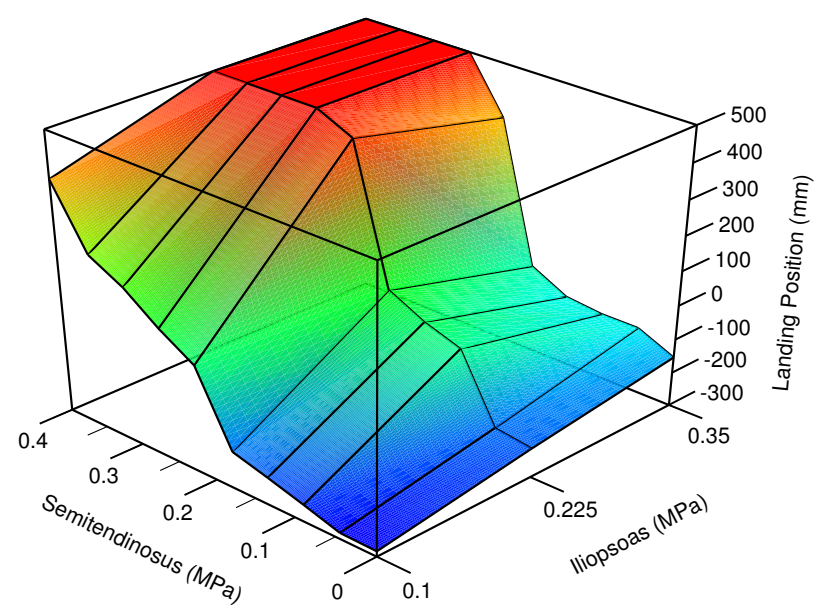

Fig. 5. Relationship between iliopsoas and semitendinosus during hopping.

precise, since the hindlimb's height is specially affected by the pressure at GA.

For the second phase of our experiment we used data acquired from the first phase and controlled a hopping hindlimb, following a target value during 72 sequential jumps.

\section{RESULTS}

Initial data acquired from hopping shows the behavior of muscles during one hopping. Pressure inside of each muscle was recorded at a sampling rate of $40 \mathrm{~Hz}$, where the hindlimb takes off, reaches apex and lands, preparing for the next jump, as seen in Fig. 4. Pressure at GA, ST and IL were $0.33,0.18$ and $0.25 \mathrm{MPa}$, respectively.

Systematically tuning pressure values for IL and ST, both responsible for rotating the hip, and averaging the results of 25 sequential jumps for each configuration, we plotted a tridimensional graph, as seen in Fig. 5. The adopted pressure for GA at these tests was $0.33 \mathrm{MPa}$. Any landing position above $200 \mathrm{~mm}$ or below $-200 \mathrm{~mm}$ should be understood as

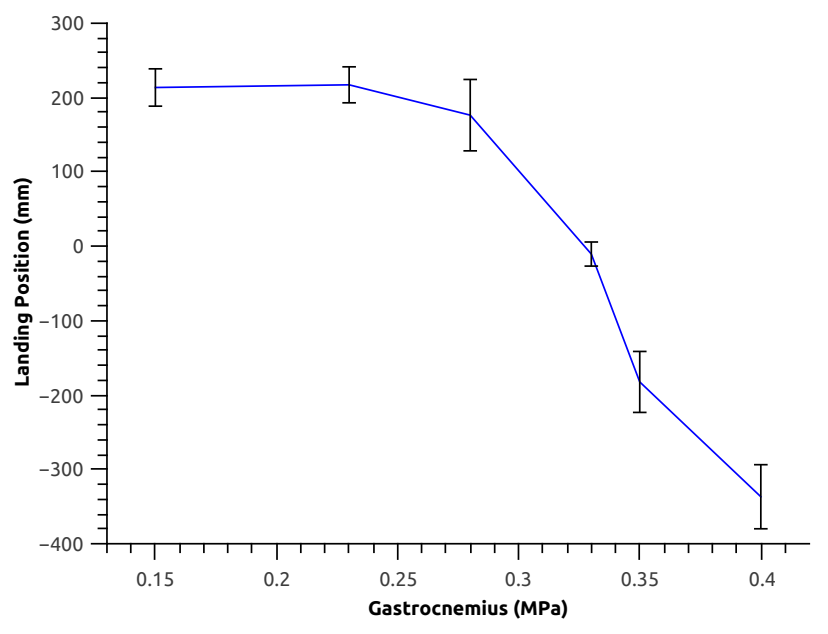

Fig. 6. Landing position during hopping with different GA settings.

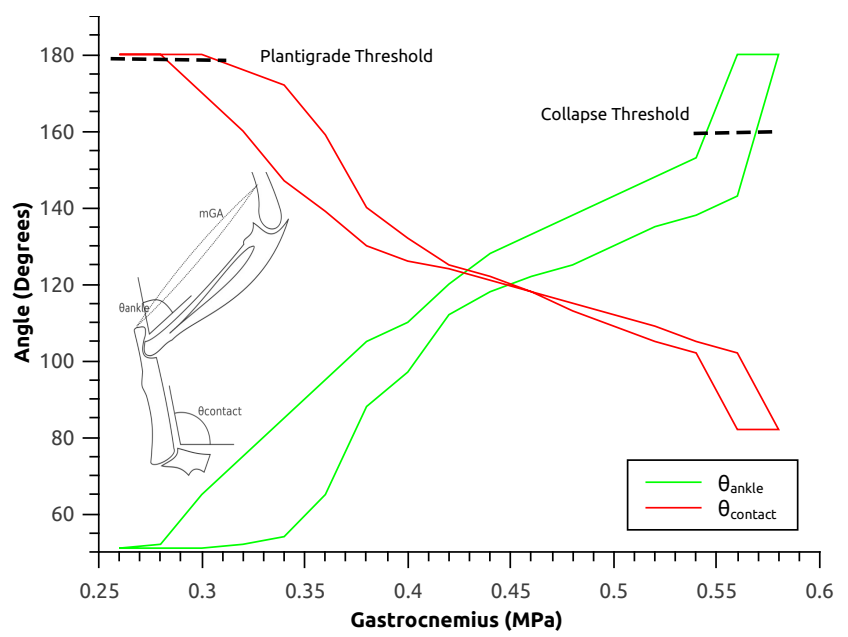

Fig. 7. Angles and hysteresis for ankle and ground contact for GA changes.

the limb climbing the slopes.

After comparative data between IL and ST, the influence of gastrocnemius on directional change was measured. Setting IL and ST pressures at $0.25 \mathrm{MPa}$ and $0.15 \mathrm{MPa}$, respectively, we calculated the average and standard deviation of 25 jumps for 6 different pressures at GA, as shown in Fig. 6 . Following this experiment, we measured the contribution of GA pressure to angle variation at ankle and ground contact, as seen in Fig. 7.

The left dashed line at the graph indicate the point where the transition from digitigrade to plantigrade stance happens, while the right dashed line indicates angles where the hindlimb collapses. The criteria for defining these two thresholds is when the ankle touches the floor and when the angle at the ankle reaches 160 degrees, respectively.

Using data from previous figures, we estimated a relationship between ST pressure and landing position. Owing to better results compared to the polynomial approach, we decided to use a Boltzmann based correlation equation of the available data set: 


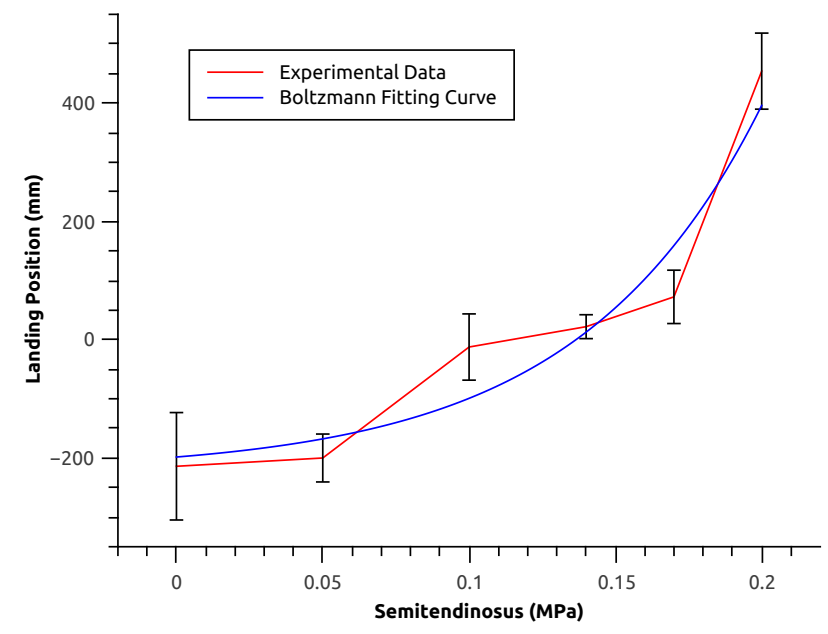

Fig. 8. Boltzmann fitting defining relationship between semitendinosus and landing position.

$$
X_{\left(p_{S T}\right)}=A_{2}+\frac{A_{1}-A_{2}}{1+e^{\left(p_{S T}-p_{S T} 0\right) / d p_{S T}}}
$$

where $X_{p_{S T}}$ is the landing position, $A_{1}$ the lower limit, $A_{2}$ the upper limit, $p_{S T} 0$ is approximately half of the pressure amplitude and $d p_{S T}$ is the pressure range. The plotted curve can be found in Fig. 8. For the actual experiment, a lookup table relating pressures and landing positions was used, simplifying the control method and avoiding logaritmics at programming level.

Finally, using this same curve we performed a sequential jumping experiment, changing landing position parameters. Results for this final experiment can be found in Fig. 9, where we perform 72 jumps, defined arbitrarily. The two horizontal dashed lines indicate the points where the foot lands on the slope.

\section{DISCUSSION}

The main objective of this work is to give a roboticist approach on a feline hindlimb muscular role during hopping. Analyzing pressure data from Fig. 4 and comparing with the activation pattern (Fig. 2), we can notice that the contraction of $\mathrm{AB}$ (hip extensor) seems to increase the tension of its antagonist, IL (hip flexor). The importance of IL before the beginning of the jump in our robotic hindlimb is to keep the hip joint in a centered position, where less pressure would result in a standing stance with the body slightly tilted backwards.

\section{A. Iliopsoas and Semitendinosus Roles}

Combining this pressure data with the relationship between IL and ST (Fig. 5), we can conclude that increasing the pressure of either muscle results in a positive foot placement. While a higher contraction on iliopsoas naturally brings the foot forward, since this muscle is the hip flexor, we observed that the increase in semitendinosus pressure rotates the hip backwards, thus bringing the center of gravity to the back and inducing sagittal rotation of the full system in mid-air.

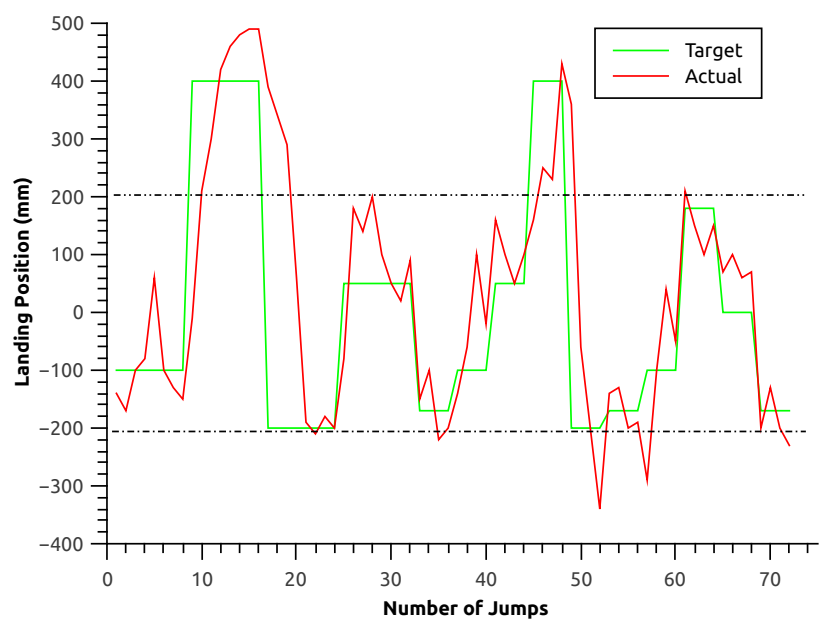

Fig. 9. Jumping experiment with 72 sequential jumps and their target value.

While hopping with ST pressures greater than 0.1 $\mathrm{MPa}$ the behavior of the IL muscle was not continuous. At these pressures, higher pressures at IL did not necessarily result in crescent foot placement. We speculate that at high IL pressures $(0.35 \mathrm{MPa})$ the increase in ST pressures creates a knee flexing torque, preventing an advancement on the foot placement. With low IL pressures the same does not occur, due to the lower hip flexion torque.

\section{B. Gastrocnemius Role}

On the GA muscle, the opposite phenomenon was observed. Increasing GA pressure resulted in a negative foot placement, and we suggest that the extension of the ankle combined with the flexion of the knee brings the center of gravity of the leg to the back, as seen in Fig. 6, thus rotating the system backwards.

Although it would be possible to control the hopping direction through gastrocnemius pressure, altering gastrocnemius pressure has a critical shortcoming for the hindlimb stability: As seen in Fig. 7, different pressures for gastrocnemius result in different attack angles. At one extreme, we have a plantigrade landing, which is not very efficient dissipating potential energy from the jump, while at another we have an ankle joint locked at 180 degrees, which simplifies the leg as a 2-link system, being also degrading for energy dissipation.

\section{Control over Landing Position and Locomotion}

Although biological data on how cats change muscular activation for different jumping directions is scarce, we propose that different tension on the ST muscle largely contributes to a better control over feline leaps.

Using the Boltzmann correlation based control of semitendinosus' pressure from Fig. 8 we performed a hopping experiment. The hindlimb sequentially hopped 72 times, tracking a target value for landing position, as seen in Fig. 9. The error present in this method is a strong indicative that, combined with the ST tonal difference, other muscles could 
also be co-activated, hence the highly complex biological structure.

This approach widens our view for the possibility of correlating jumping direction control with specific gait patterns, such as pronking and bounding. The possibility of using directional control on fore and hindlimbs on a quadrupedal robot to reproduce these gait patterns would possibly make robot locomotion easier.

\section{Study Limitations}

In this paper we proposed a biomimetic hindlimb, using artificial muscles and adopting biologic dimensions. While we used a vertical jump as a testing method, biological locomotion is way more complex than this movement, merely functioning as a simplified experiment to test the leg. Future publications will adopt the same structure on a wider range of motions, validating the hindlimb in different aspects of animal locomotion.

\section{CONCLUSIONS AND FUTURE WORKS}

The morphological complexity of animals is a conundrum which roboticists are still scratching the surface, and this work delves into the problem aiming to provide more clarity on the subject.

We built a biomimetic hindlimb with an experimental platform for hopping. After creating a muscular pattern for vertical jumping, we systematically tried different pressure configurations for monoarticular and biarticular air muscles, finding a relationship between muscular activation and landing position in a series of jumps. Although gastrocnemius, iliopsoas and semitendinosus contribute to the sagittal rotation, the most reliable way for changing hopping direction was shown to be through semitendinosus tonus control.

We performed experiments applying the proposed tonus control, and the proposed method may offer a possible explanation for a directional change of a real feline jump, and the result could be extended to gait pattern explanation, such as bouncing and pronking gait, where the average speed could be controlled by semitendinosus activation.

Future works will focus on reproducing phenomena observed on feline-related publications [19] [20], associating muscular activation to air pressure, hoping to provide a constructivist feedback to biologists.

Integration of the hindlimb with the full body Pneupard will provide us with a full biomimetic platform, being the closest robotic platform to date to mimic the Felidae biological system (considering morphology, muscular redundancy or number of active muscles). This platform will be used to validate the aforementioned controlling method, aiming to control Pneupard direction during locomotion.

\section{REFERENCES}

[1] I. Engberg and A. Lundberg, "An electromyographic analysis of muscular activity in the hindlimb of the cat during unrestrained locomotion", Acta Physiol. Scand., Vol. 75, 1969, pp. 614-630.

[2] G.E. Goslow Jr., R.M. Reinking and D.G. Stuart, "The cat step cycle: hind limb joint angles and muscle lengths during unrestrained locomotion", J. Morphol., Vol. 141, 1973, pp. 1-42.

[3] S. Rasmussen, A.K. Chan and G.E. Goslow Jr., "The cat step cycle: electromyographic patterns for hindlimb muscles during posture and unrestrained locomotion", J. Morphol., Vol. 155, 1978, pp. 253-269.

[4] A. English, "An electromyographic analysis of forelimb muscles during overground stepping in the cat", J. Exp. Biol., Vol. 76, 1978, pp. 105-122.

[5] B.I. Prilutsky, W. Herzog and T.L. Allinger, "Mechanical power and work of cat soleus, gastrocnemius and plantaris muscle during locomotion: possible functional significance of muscle design and force patterns", J. Exp. Biol., Vol. 199, 1996, pp. 801-814.

[6] B.I. Prilutsky, W. Herzog and T. Leonard, "Transfer of mechanical energy between ankle and knee joints by gastrocnemius and plantaris muscles during cat locomotion", J. Biomech., Vol. 29, 1996, pp. 391403.

[7] G.J. van Ingen Schenau, M.F. Bobbert and R.H. Rozendal, "The unique action of bi-articular muscles in complex movements", J. Anat., Vol. 155, 1987, pp. 1-5.

[8] S. Gielen, G.J. van Ingen Schenau, T. Tax and M. Theeuwen, "The activation of mono- and bi-articular muscles in multi-joint movements", Multiple Muscle Systems: Biomechanics and Movement Organization, Springer Verlag, New York, 1990.

[9] K. Hosoda, Y. Sakaguchi, H. Takayama and T. Takuma, "Pneumaticdriven jumping robot with anthropomorphic muscular skeleton structure", Auton. Robot., Vol. 28, 2010, pp. 307-316.

[10] Y. Fukuoka, H. Kimura and A.H. Cohen, ”Adaptive dynamic walking of a quadruped robot on irregular terrain based on biological concepts", J. Robot. Res., Vol. 22, 2003 , pp. 187-202.

[11] A. Sproewitz, A. J.Ijspeert et al., "Oncilla robot : a light-weight bioinspired quadruped robot for fast locomotion in rough terrain", in Proc. Fifth Intl. Symp. Adaptive Motion on Animals and Machines, Osaka, Japan, 2011.

[12] K. Aschenbeck, N. Kern, R. Bachmann and R. Quinn, ”Design of a quadruped robot driven by air muscles" in Proc. Intl. Conf. Biomed. Robot. and Biomechatronics, 2006, pp. 875-880.

[13] K. Tsujita, T. Kobayashi, T. Inoura and T. Masuda, "Gait transition by tuning muscle tones using pneumatic actuators in quadruped locomotion", in Proc. Intl. Conf. on Intelligent Robots and Systems, 2008, pp. 2453-2458.

[14] M. Lewisy, M. Buntingy, B. Salemi and H. Hoffmann, "Toward ultra high speed locomotors: design and test of a cheetah robot hind limb", in Proc. Intl. Conf. on Robotics and Automation, 2011, pp. 1990-1996.

[15] S.H. Hyon, "Development of a biologically inspired hopping robot Kenken", in Proc. Intl. Conf. on Robotics and Automation, 2002, pp. 3984-3991.

[16] O. Ekeberg and K. Pearson, "Computer simulation of stepping in the hind legs of the cat: an examination of mechanisms regulating the stance-to-swing transition", J. Neurophysiol., Vol. 94, 2005 , pp. 42564268.

[17] R. Niiyama, S. Nishikawa and Y. Kuniyoshi, "Athlete Robot with applied human muscle activation patterns for bipedal running", in Proc. Intl. Conf. Humanoids Robots, 2010, pp. 498-503.

[18] I. Boblan, "A human-like robot hand and arm with fluidic muscles: biologically inspired construction and functionality", Embodied Artficial Intelligence, Springer, Dagstuhl Castle, 2004.

[19] L.M. Day and B.C. Jayne, "Interspecific scaling of the morphology and posture of the limbs during locomotion of cats (Felidae)", J. Exp. Biol., Vol. 210, 2007, pp. 642-654.

[20] T.J. Burkholder and T.R. Nichols, "Three-dimensional model of the feline hindlimb", J. Morphol., Vol. 261, 2004, pp. 118-129. 